\title{
X-chromosome Inactivation in American Mink iPSCs
}

\author{
Inna Pristyazhnyuk \\ Sector of Genomic Mechanisms of \\ Ontogenesis \\ Institute of Cytology and Genetics \\ SB RAS \\ Novosibirsk, Russia \\ iprist@mail.ru
}

\author{
Aleksei Menzorov \\ Sector of Cell Collections \\ Institute of Cytology and Genetics \\ SB RAS \\ Novosibirsk, Russia \\ menzorov@bionet.nsc.ru
}

\begin{abstract}
Induced pluripotent stem cells (iPSCs) were produced from many species. One of the key iPSC characteristics is $\mathrm{X}$-chromosome inactivation status. Both active $X$-chromosomes mark naïve pluripotency, the presence of inactive X-chromosome suggests primed pluripotency. We had previously shown that American mink embryonic stem cells have both $\mathrm{X}$-chromosomes active. In this study, we produced American mink iPSCs from XX embryonic fibroblasts and studied $\mathrm{X}$-chromosome inactivation. We performed immunocytochemical staining of $H 3 K 27 \mathrm{me} 3$ chromatin modification (marker of inactive chromatin) and reverse transcription PCR of the Xist expression (marker of inactivated $\mathrm{X}$-chromosome) in single cells. We have shown that according to the immunocytochemical analysis of three iPSC lines, from 56.8 to $95.6 \%$ of cells had both $\mathrm{X}$-chromosome homologs active. Xist expression data confirmed these results for one iPSC line, but unexpectedly the majority of cells in the other two iPSC lines were positive for Xist expression, so one of the X-chromosomes was presumably inactivated. Thus, we analyzed $X$-chromosome inactivation in XX American mink iPSCs for the first time and revealed the presence of different $\mathrm{X}$-chromosome inactivation patterns.
\end{abstract}

Keywords - induced pluripotent stem cells, X-chromosome inactivation, American mink, Neovison vison, Xist

\section{Introduction}

Induced pluripotent stem cells (iPSCs) share similar properties with embryonic stem cells and allow studying early embryonic development of species whose biological material is difficult to obtain. Currently, iPSCs have been produced for less than 20 species. Among order Carnivora, there are just a few species: snow leopard, dog, cat, and American mink. One of the important pluripotency markers is X-chromosome. Naïve pluripotent stem cells have both X-chromosome homologs active (XaXa) and primed - one inactivated homolog (XaXi). We have previously shown that American mink (Neovison vison) embryonic stem cells have both active $\mathrm{X}$-chromosomes $[1 ; 2]$. In this study, we analyzed $\mathrm{X}$ chromosome status in American mink iPSCs produced from $\mathrm{XX}$ embryonic fibroblasts.

\section{Materials and Methods}

American mink iPSC lines iNV1XX1, iNV2XX5, and iNV5XX2 were produced from XX embryonic fibroblasts NV1, NV2, and NV5, respectively, according to previously published protocol [1] with minor modifications. Cell culture medium contained $10 \%$ fetal bovine serum and $10 \%$ KnockOut Serum Replacement (Thermo Fisher Scientific, USA) during iPSC derivation, after third passage $20 \%$ fetal bovine serum was used.
X-chromosome inactivation was estimated by immunocytochemistry and single-cell reverse transcription (RT) PCR.

One of the markers of inactive X-linked chromatin modifications is histone $\mathrm{H} 3$ trimethylated at lysine 27 (H3K27me3). Cells were stained for H3K27me3 according to the previously published protocol [1]. H3K27me3 signal was interpreted as the presence of the inactive X-chromosome.

For RT-PCR analysis, we manually collected individual cells into lysis buffer and synthesized cDNA in $5 \mu \mathrm{l}$ volume using M-MuLV-RH Reverse Transcription Kit (Biolabmix, Russia) according to the manufacturer's recommendations. Then, $2 \mu \mathrm{l}$ of cDNA solution were used for PCR using BioMaster HS-Taq PCR-Color kit (Biolabmix, Russia) according to the manufacturer's recommendations. Primer pairs for Xist and Rex 1 amplification were located in different exons, so DNAseI treatment of RNA was not necessary.

\section{Results}

iPSCs had normal diploid karyotype (30, XX), expressed pluripotency marker genes Oct4, Rexl, and as expected had practically no expression of Nanog.

We assumed that the majority of iPSCs would be XaXa with Xist-/Rex $1+$ pattern, where the absence of Xist transcript means lack of $\mathrm{Xi}$, and the presence of Rexl serves as a pluripotency marker.

Immunochemical analysis of $\mathrm{H} 3 \mathrm{~K} 27 \mathrm{me} 3$ was verified on $\mathrm{XX}$ and XY embryonic fibroblasts. XX NV1 and NV5 embryonic fibroblasts showed that from 84.9 to $86.7 \%$ of cells are $\mathrm{XaXi}$, as one of the $\mathrm{X}$-chromosomes is inactivated in somatic cells. All cells of XY NV4 fibroblasts lacked Xi, as expected. Analysis of 77-89 cells for each iPSC line revealed that from 56.8 to $95.6 \%$ of cells had both X-chromosome homologs active (Fig. 1).

To verify RT-PCR method we analyzed the expression of Xist transcripts in 20 individual embryonic fibroblast cells. 19 XX NV5 cells had Xist transcript marking Xi, none XY NV4 cells had it, as expected. Then we analyzed the number of Xist$/$ Rex + cells in iPSCs. It varied from 0 to $30.8 \%$ (Fig. 1). The number of Xist-/Rex + cells was $30.8 \%$ for iNV1XX1 which is comparable with $45.7 \%$ of XaXa cells. As for iNV2XX5 and iNV5XX2, there was just one Xist-/Rex+ cell, the majority of cells were pluripotent with $\mathrm{XaXi}$ status $(X i s t+/ \operatorname{Rex} 1+)$ or differentiated (Xist+/Rex1-). 
A

Percentage of cells with different Xist/Rex1 expression patterns

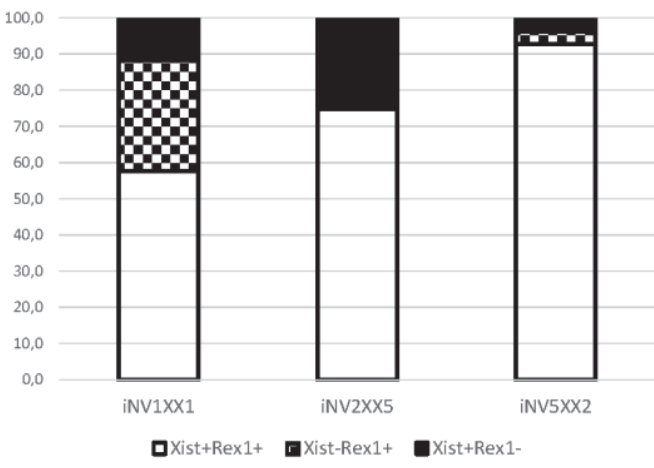

B Percentage of cells with different $\mathrm{X}$-chromosome inactivation patterns

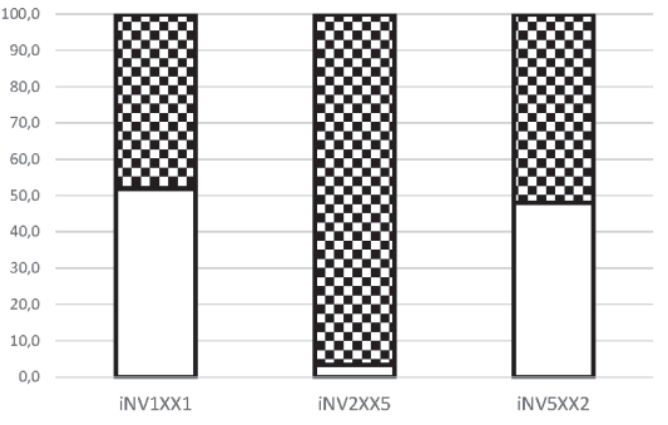

Fig. 1. X-chromosome inactivation patterns in $\mathrm{XX}$ American mink iPSCs.

\section{Discussion}

Mink embryonic stem cells have both X-chromosomes active $[1 ; 2]$. We expected that iPSCs would have XaXa status, as iPSCs are considered practically identical to ES cells. The immunochemical analysis was consistent with that; the majority of cells were $\mathrm{XaXa}$, and some $\mathrm{XaXi}$ cells were expected, as there is a certain percentage of differentiated pluripotent stem cells in in vitro culture. RT-PCR analysis of single cells had given different results.

The discordance between immunocytochemical and RTPCR analyses could have several reasons. First, the iPSCs could have changed XaXa status to XaXi without any changes in morphology and/or gene expression. It is known that cell density, duration of in vitro culture, and other factors could promote pluripotent stem cell differentiation. Second, though Xist is a well-known X-chromosome inactivation marker, its expression is one of the first steps of the X-chromosome inactivation process. It is possible, that there is an onset of Xist expression in XaXa cells that is revealed by RT-PCR but does not coincide with $\mathrm{H} 3 \mathrm{~K} 27 \mathrm{me} 3$ chromatin modification, as it has not started yet. Third, there might be a certain threshold of Xist and other genes expression to trigger $\mathrm{X}$-chromosome inactivation [3]. We were not able to quantify the level of Xist expression, so the presence of Xist transcript may not be enough to conclude $\mathrm{X}$-chromosome inactivation.

Overall, we produced American mink iPSCs from XX embryonic fibroblasts for the first time. Our data show that iPSCs could have $\mathrm{XaXa}$ state of $\mathrm{X}$-chromosomes that is one of the naïve pluripotency markers or could have XaXi state that is either marker of primed pluripotency or sign of differentiation.

\section{ACKNOWLEDGMENT}

The reported study was funded by RFBR according to the research project No. 20-04-00369 and by the Ministry of Education and Science of Russia, state project No. 0324-20190041-C-01. Cell lines are available at the Collective Center of ICG SB RAS "Collection of Pluripotent Human and Mammalian Cell Cultures for Biological and Biomedical Research" (http://ckp.icgen.ru/cells/; http://www.biores.cytogen.ru/icg_sb_ras_cell/).

\section{REFERENCES}

[1] A. G. Menzorov, N. M. Matveeva, M. N. Markakis, V. S. Fishman, K. Christensen et al., "Comparison of American mink embryonic stem and induced pluripotent stem cell transcriptomes," BMC Genomics, vol. 16(Suppl 13), S6, December 2015.

[2] M. A. Sukoyan, S. Y. Vatolin, A. N. Golubitsa, A. I. Zhelezova, L. A. Semenova, O. L. Serov, "Embryonic stem cells derived from morulae, inner cell mass, and blastocysts of mink: comparisons of their pluripotencies," Mol Reprod Dev., vol. 36(2), pp. 148-158, October 1993.

[3] T. S. Barakat, I. Jonkers, K. Monkhorst, J. Gribnau, "X-changing information on X inactivation," Exp Cell Res., vol. 316(5), pp. 679687, March 2010 